\title{
Estudo da glibenclamida no tratamento do diabete melito gestacional e sua repercussão no peso e glicemianeonatal
}

Study of glibenclamide for the management of gestational diabetes mellitus and its impact on neonatal weight and glucose levels

Autor: Jean Carl Silva

Orientadora: Profa. Dra. Anna Maria Bertini

Co-orientador: Prof.Dr. Dr. Wladimir Taborda

Tese de Doutorado apresentada ao Departamento de Obstetrícia da Universidade Federal de São Paulo - UNIFESP, em 16 de agosto de 2005.

Objetivos: estudar a glibenclamida no tratamento do diabete melito gestacional (DMG) e sua repercussão no peso e glicemia do recém-nascido, em comparação com a insulina. Material e Métodos: ensaio clínico randomizado, realizado no período entre $1^{\circ}$ de outubro de 2003 e 8 de março de 2005. Foram sujeitos deste estudo 72 gestantes com DMG que necessitaram de terapêutica complementar a dietoterapia e atividade física, randomizadas e alocadas em dois grupos com estratégias terapêticas diferentes, insulina e glibenclamida. Resultados: as características gerais nos grupos não tiveram diferença, com exceção dos resultados do OGTT $75 \mathrm{~g}$ que apresentaram valores maiores no grupo da glibenclamida $(p=0,02)$. As glicemias maternas coletadas em jejum e pós-prandial não apresentaram diferença. Seis $(18,75 \%)$ gestantes atingiram a dose máxima de glibenclamida sem o controle glicêmico. O peso dos recém-nascidos foi maior no grupo da glibenclamida $(p=0,01)$, com peso médio neste grupo de $3.372 \mathrm{~g}$, contra 3.082g no grupo da insulina. Não houve diferença na incidência de recém-nascidos GIG, porém encontramos diferença $(p=0,01)$ na incidência de macrossômico entre os grupos da glibenclamida e insulina (15,6\% e 0\%, respectivamente). A hipoglicemia neonatal estava mais presente $(p=0,01)$ nos recém-nascidos do grupo da glibenclamida $(25 \%)$ que com insulina $(2,7 \%)$, mas tivemos apenas um caso de hipoglicemia neonatal persistente, que necessitou infusão endovenosa de glicose, no grupo da glibenclamida. Conclusão: a glibenclamida pode ser a droga de escolha para tratamento do DMG na maioria das pacientes. $O$ peso dos recém-nascidos e a incidência de hipoglicemia foi maior no grupo da glibenclamida, porém o peso médio foi normal e tivemos apenas um caso de hipoglicemia neonatal que necessitou de glicose endovenosa.

PALAVRAS-CHAVE: Diabete melito gestacional, Glibenclamida, Recém-nascido.

\section{Fatores associados à infecção do sítio cirúrgico após cirurgia para o tratamento do câncer de mama em mulheres usuárias do sistema de drenagem}

Factors associated with infection of the surgical site after surgery for the treatment of breast cancer in women using the drainage system

Autora: Wilza Andrade Barbosa Felippe

Orientador: Prof. Dr. Guilherme Loureiro Werneck

Co-Orientador: Prof. Dr. Guilherme Santoro Lopes

Dissertação de Mestrado submetida ao Programa de Pós-Graduação (em Saúde Coletiva), na Área de Epidemiologia e Bioestatística, no Núcleo de Estudos de Saúde Coletiva - NESC, da Universidade Federal do Rio de Janeiro UFRJ, em 2 de junho de 2005.

Introdução: a infecção do sítio cirúrgico ISC após o tratamento cirúrgico para o câncer de mama além de expor a mulher ao uso de antimicrobianos, pode muitas vezes adiar o início do tratamento adjuvante e gerar prejuízos sociais inseridos no seu processo de recuperação e reabilitação. Objetivo: estudar os fatores associados à ocorrência da ISC em mulheres submetidas ao tratamento cirúrgico para o câncer de mama com linfadenectomia axilar e usuárias do sistema de drenagem. Metodologia: Foi realizado um estudo prospectivo com 354 mulheres submetidas ao tratamento cirúrgico para o câncer de mama com linfadenectomia axilar no Hospital do Câncer III / INCA - MS, no período de março a julho de 2004. A coleta de dados ocorreu durante a assistência direta prestada às pacien- tes no ambulatório de egressos e complementadas pela análise do prontuário hospitalar. Foi realizada a análise multivariada por regressão log-binomial para identificar as variáveis independentemente associadas com ISC. Resultados: a incidência cumulativa de ISC foi de $16,9 \%$. ISC não foi significativamente influenciada pelo tempo de permanência do dreno. Conclusão: No modelo final, observou-se que idade, necrose tecidual e a colonização do sistema de drenagem configuraram o conjunto de fatores associados para ISC após cirurgia para o tratamento do câncer de mama com linfadenectomia axilar.

PALAVRAS-CHAVE: Câncer de mama; Linfadenectomia axilar; Infecção cirúrgica; Sistema de drenagem 